\begin{tabular}{|c|l|}
\hline Title & Spatial light modulator with an over-two-octave bandwidth from ultraviolet to near infrared \\
\hline Author(s) & Hazu, Kouji; Sekikawa, Taro; Y amashita, Mikio \\
\hline Citation & $\begin{array}{l}\text { Optics Letters, 32(22), 3318.3320 } \\
\text { https:/doi.org/L0.1364/0L.32.003318 }\end{array}$ \\
\hline Issue Date & 2007-11-15 \\
\hline Doc URL & http://hdl.handle.net/2115/45352 \\
\hline Rights & ○ 2007 Optical Society of A merica \\
\hline Type & article \\
\hline File Information & OL 32-22_3318-3320.pdf \\
\hline
\end{tabular}

Instructions for use 


\title{
Spatial light modulator with an over-two-octave bandwidth from ultraviolet to near infrared
}

\author{
Kouji Hazu,* Taro Sekikawa, and Mikio Yamashita \\ Department of Applied Physics, Hokkaido University, and Japan Science and Technology Agency, CREST, \\ Kita-Ku, Sapporo 060-8628, Japan \\ *Corresponding author: kouji@eng.hokudai.ac.jp
}

Received August 28, 2007; revised October 12, 2007; accepted October 12, 2007; posted October 18, 2007 (Doc. ID 86557); published November 6, 2007

We developed a 1-pixel ultraviolet-to-near-infrared (UV-to-NIR) liquid-crystal spatial light modulator (LCSLM) and clarified its phase modulation properties in detail, for the first time to our knowledge. The employed liquid crystal is transparent over $260-1100 \mathrm{~nm}$. A phase modulation capability of $55.8 \mathrm{rad}$ at $305 \mathrm{~nm}$ and $14.0 \mathrm{rad}$ at $1000 \mathrm{~nm}$ is enough to compensate for UV-to-NIR nonlinear chirped pulses. The LC-SLM driving parameters of a period $T=13 \mathrm{~ms}$ and an applied voltage $V_{\mathrm{DD}}=7.0 \mathrm{~V}$ were determined. The 648-pixel extension of this new device will permit us to realize the high-power generation of single subcycle optical pulses and the direct UV-to-NIR pulse shaping. (C) 2007 Optical Society of America

OCIS codes: $320.0320,320.5520,320.5540,320.7080,320.7160$.

Recently, $2.8 \mathrm{fs}$ single isolated optical pulses in the monocycle region were generated by means of feedback chirp compensation of pulses ultrabroadened by self-phase modulation (SPM), where a liquid-crystal spatial light modulator (LC-SLM) [1] was employed [2]. More recently, $2.6 \mathrm{fs}, 1.3$ cycle pulses (1.4 GW peak power) were generated by using the same compensation technique for pulses ultrabroadened by induced-phase modulation (IPM) as well as SPM [3]. Although the pulse spectrum has been broadened over the octave ranging from 300 to $1000 \mathrm{~nm}$ by IPM as well as SPM [4], which corresponds to $1.5 \mathrm{fs}$, 0.9 cycle transform-limited pulses [4], further shortpulse compensation has not succeeded owing to the bandwidth limitation resulting from the ultraviolet (UV) absorption by the liquid crystal. Therefore, the LC-SLM operating over the UV-to-near-infrared (UVto-NIR) range is strongly desired. Moreover, the twodimension-like LC-SLM operating in the similar wavelength region is required as a chirp compensator with an effectively high optical-damage threshold for the realization of a monocycle, high-powered optical source. On the other hand, the LC-SLM has been widely used as a programmable pulse shaper for quantum control of atoms and molecules [5]. However, the conventional LC-SLM cannot be applied for quantum control of important organic and biological molecules because those molecules have absorption bands in the UV region. The LC-SLM has also been used for holographic memory, microscopic optical imaging, and three-dimensional femtosecond laser processing. For further progress in those fields, the UVLC-SLM is very useful as an accurate wavefront phase controller.

There are many other chirp compensators such as a chirped mirror (CM) [6], an acousto-optic modulator (AOM) [7], a mechanically deformable mirror (DM) [8], and a mechanically controllable micromirror SLM (MM-SLM) [9]. Recently, a 1.5-octave CM pair has been demonstrated [6]. Because of fixed disper- sion characteristics, a CM pair cannot compensate for arbitrary nonlinear chirp. In addition, it has a low limitation value for the amount of chirp to be compensated. Furthermore, a CM cannot be used for pulse shaping. The AOM, the DM, and the MM-SLM are programmable phase compensators. However, the AOM has a limitation for the compressed pulse duration of about $10 \mathrm{fs}$ due to the highly dispersive AOM crystal [7]. The DM has a low spatial resolution because of the low piezo stacks, compared with 648 pixels of the LC-SLM. The MM-SLM has an operation wavelength range of $200-900 \mathrm{~nm}$ but a relatively high ratio of gap size to pixel size and low phase resolution [9].

In this Letter, we demonstrate a 1-pixel LC-SLM offering high transmission and enough phase modulation over a 2-octave bandwidth from UV to NIR for what we believe to be the first time. This technology will become instrumental in generating subcycle, high-powered pulses and controlling temporal electric fields directly over the entire region from UV to NIR.

Up to now, in general, a mixture of cyano group derivatives has been employed as an LC for a traditional visible LC-SLM. Instead, we used a mixture of cyclohexane derivatives having fluorine substituents as a nematic LC (UV-LC), which was supplied from Chisso Petrochemical Corporation. Its optical and dielectric anisotropies are 0.080 (the extraordinary refractive index $n_{e}=1.485$ ) at $589 \mathrm{~nm}$ and 4.7 (the dielectric constant of the minor axis direction $\varepsilon_{\perp}=3.7$ ) at $1 \mathrm{kHz}$, respectively. Figure 1(a) shows the UVvisible-NIR transmittance curves of the two LCs. The new UV-LC shows the high transmittance of $92.2 \%, 90.6 \%$, and $94.0 \%$ at 260,800 , and $1100 \mathrm{~nm}$, respectively, under the condition of the $30 \mu \mathrm{m}$ thick cell structure [Fig. 1(b)], compared with the traditional visible $\mathrm{LC}$ of $0.0 \%, 87.4 \%$, and $82.9 \%[2,3]$.

We fabricated a 1-pixel LC-SLM that was rectangular with a dimension of $10 \mathrm{~mm} \times 10 \mathrm{~mm}$ and a 


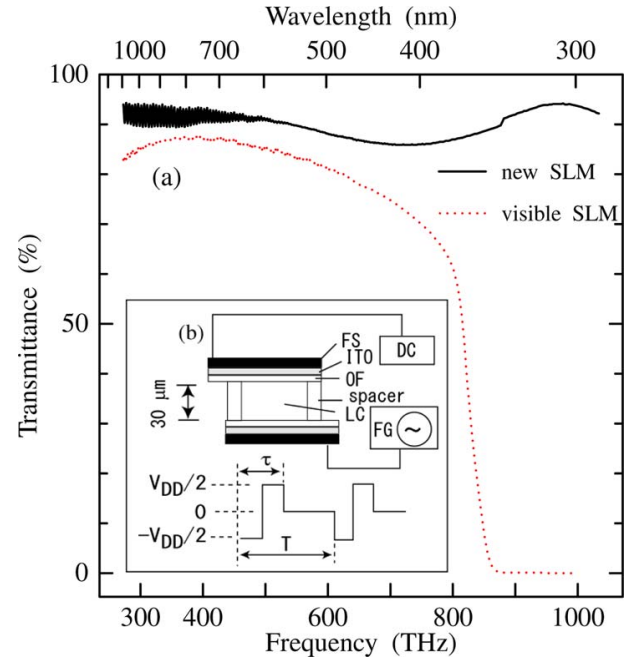

Fig. 1. (Color online) (a) Transmittance curves of UV-LCSLM (solid curve) and visible LC-SLM (dotted curve). (b) Structure of UV-LC-SLM and applied voltage waveform. LC, liquid crystal; FS, fused silica substrate; ITO, indium tin oxide film; OF, oriented organic film; DC, DC power source; FG, function generator; T, period; $\tau$, pulse width.

$30 \mu \mathrm{m}$ cell thickness. The cell is uniformly thick because spacers of a highly precise size were sprayed onto the surface. The UV-LC was sandwiched between two fused silica substrates (1 mm thickness). Indium tin oxides and oriented organic films were deposited on the fused silica substrates [Fig. 1(b)]. The orientation of the UV-LC was parallel.

We applied the AC-like waveform (its period $T$ ) with an amplitude of $V_{\mathrm{DD}}$ to one of the substrates of the new UV-LC-SLM and applied the DC voltage of $V_{\mathrm{DD}} / 2$ to the other [Fig. 1(b)]. The AC-like voltage was applied to avoid the degradation of the UV-LC owing to the attachment of ion contaminants to the surfaces in the UV-LC $[2,3]$. In order to compensate for chirped pulses with an arbitrary spectral phase over the UV-to-NIR region, a phase modulation $\Delta \phi$ of at least $2 \pi$ rad at the longest wavelength of $1100 \mathrm{~nm}$ is needed. Moreover, for accurate compensation, the precise control of the pulse width $\tau$ of the applied voltage [see Fig. 1(b)] is requested (with an accuracy of 12 bits) because the phase shift is controlled by the pulse width $\tau$ (not by the applied voltage $V_{\mathrm{DD}}$ ) [2,3] Accordingly, phase modulation properties at different wavelengths $(\lambda)$ from UV to NIR were investigated in detail by using a channeled spectrum technique [10] so as to determine the values of the parameters of $T$ and $V_{\mathrm{DD}}$.

The employed apparatus was similar to [10] except for the following points. The UV-LC-SLM was placed between a polarizer and an analyzer that had a crossed Nicol configuration and was rotated by $45^{\circ}$ from the principal axes of the UV-LC. At $400 \mathrm{~nm}$, we exchanged light sources (a Xe lamp for the longer wavelength region and a halogen lamp for the shorter one), monochromators ( $0.5 \mathrm{~m}$ focal length, one with a 1200 groove $/ \mathrm{mm}$ grating and $0.3 \mathrm{~m}$ focal length, one with a 150 groove $/ \mathrm{mm}$ grating), and detectors (the intensified charged coupled device [intensified $\mathrm{CCD}$ ] and the CCD). A $15 \mathrm{~mm}$ thick retardation plate was used to get a clear interference of the transmitted light.

Figure 2(a) shows the period dependence of the phase modulation at $V_{\mathrm{DD}}=6 \mathrm{~V}, \lambda=1000 \mathrm{~nm}$, and $\tau / T$ $=0.5$. The applied voltage of $V_{\mathrm{DD}}=6 \mathrm{~V}$ was selected as a value close to that of the visible LC-SLM, which we had previously used $\left(V_{\mathrm{DD}}=5.0 \mathrm{~V}, T=2.7 \mathrm{~ms}\right)[2,3]$. The modulation increases rapidly and reaches the maximum value of $13.1 \mathrm{rad}$ at $T=13 \mathrm{~ms}$ and then decreases. Therefore, we decided that the suitable pe$\operatorname{riod} T$ is $13 \mathrm{~ms}$.

Next, we decided the optimum applied voltage. To get a large and stable phase modulation for chirp compensation, one had better drive the UV-LC-SLM at the saturation voltage region. Figure 3(a) shows the applied voltage dependence of the phase modulation at $\tau=6.5 \mathrm{~ms}, T=13 \mathrm{~ms}$, and $\lambda=305,600$, and $1000 \mathrm{~nm}$. The phase modulation saturated at $V_{\mathrm{DD}}$ $=7.0 \mathrm{~V}$. Their modulation values were $55.8 \mathrm{rad}$ at $305 \mathrm{~nm}, 23.5 \mathrm{rad}$ at $600 \mathrm{~nm}$, and $14.0 \mathrm{rad}$ at $1000 \mathrm{~nm}$, respectively. The threshold voltage was $1.5 \mathrm{~V}$. These results suggest that the suitable applied voltage $V_{\mathrm{DD}}$ is $7.0 \mathrm{~V}$.

Furthermore, we measured the wavelength dependence of the phase modulation for different values of $\tau$ from 0.7 to $10.0 \mathrm{~ms}$ at $T=13 \mathrm{~ms}$ and $V_{\mathrm{DD}}=7.0 \mathrm{~V}$ [Fig. 3(b)]. The phase modulation values were 55.8, 22.7 , and $12.0 \mathrm{rad}$ (much larger than $2 \pi \mathrm{rad}$ ) at 305 , 600 , and $1100 \mathrm{~nm}$ under $\tau=10 \mathrm{~ms}$, respectively. This suggests that the UV-LC-SLM has the ability to compensate for highly chirped pulses with the over-2octave bandwidth from 260 to $1100 \mathrm{~nm}$. The more detailed $\tau$ dependence permits us to separate the amount $[\Delta \phi(\lambda, X)]$ of the phase modulation to a function of the gray scale $[g(X)]$ and a function of the dispersion of the UV-LC $[f(\lambda)]$. That is, $\Delta \phi(\lambda, X)$ $=f(\lambda) g(X)$. Here, $X$ is proportional to $\tau$. The $g(X)$ plays an essential part in the making of an accurate and programmable phase control by the UV-LC-SLM $[2,3]$. The obtained $f(\lambda)$ showed an excellent agreement with the UV-LC dispersion, which was measured independently.

We also measured the time-response of the UV-LCSLM using the above-mentioned interferometer. A $633 \mathrm{~nm} \mathrm{He}-\mathrm{Ne}$ laser was used as a light source. A photodiode and an oscilloscope were used for the time-response detection. The positive waveform of the voltage applied to the UV-LC-SLM was rectangu-

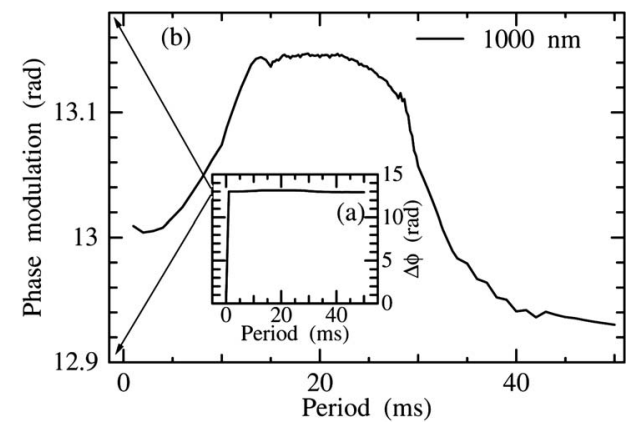

Fig. 2. (a) Period dependence of phase modulation $\Delta \phi$ at $1000 \mathrm{~nm}, V_{\mathrm{DD}}=6 \mathrm{~V}$ and $\tau / T=0.5$. (b) Enlarged y axis. 


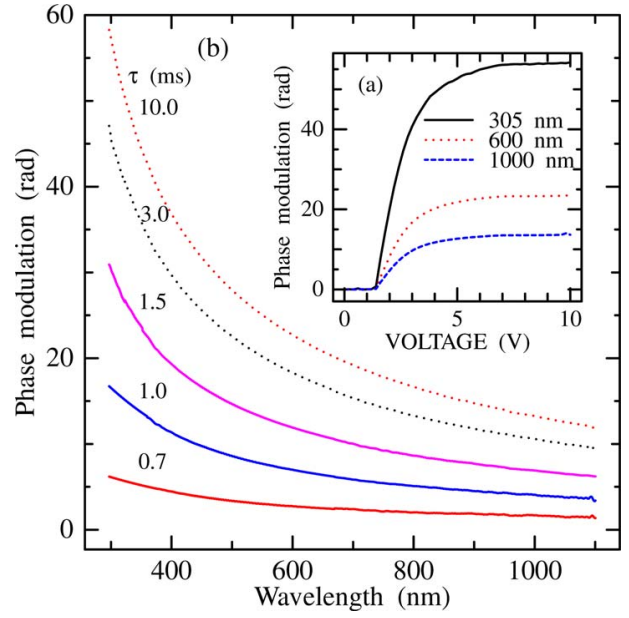

Fig. 3. (Color online) (a) Applied voltage dependence of phase modulation $\Delta \phi$ at 305 (solid curve), 600 (dotted curve), and $1000 \mathrm{~nm}$ (dashed curve) under $\tau=6.5 \mathrm{~ms}$ and $T=13 \mathrm{~ms}$. (b) Wavelength dependence of phase modulation for different widths $(\tau)$ of applied voltage pulses at $T$ $=13 \mathrm{~ms}$ and $V_{\mathrm{DD}}=7.0 \mathrm{~V}$.

lar, with a $20 \mathrm{~s}$ period and a $10 \mathrm{~s}$ pulse duration. We measured the time-dependent intensity $I(t)$ of the transmitted light as a parameter of the applied voltage of $V_{\text {appl }}$ within the range from 4 to $10 \mathrm{~V}$, which is given by

$$
I(t)=[A / 2]\left\{1-\cos \left[\varphi(t)+\varphi_{0}\right]\right\} .
$$

Here, $A$ is an intensity of incident light, $\varphi(t)$ is an induced phase modulation, and $\varphi_{0}$ is an initial phase.

Figure 4(a) shows the transient transmitted-light intensity $I(t)$ at $V_{\text {appl }}=7.0 \mathrm{~V}$. The curve $I(t)$ shows the remarkable intensity change at $t=0.176,0.232$, 0.272 , and $0.312 \mathrm{~s}$, corresponding to $\varphi(t)+\varphi_{0}=\pi, 2 \pi$, $3 \pi$, and $4 \pi$, respectively [see Eq. (1)]. Figure 4(b) shows the modulated phase $\varphi(t)$ as a function of time. The unwrapped phase was calculated using Eq. (1) assuming the continuous change with time. The transient phase change $\varphi(t)$ was slightly slow right after the voltage $V_{\text {appl }}$ was applied because the UV-LC is strongly bound by the oriented organic films at both edges. We defined the response time $\tau_{r}$ as time until the phase $\varphi(t)$ changes from 0 to $2 \pi$ rad. Figure 4(c) shows the applied voltage dependence of the response time. The response time becomes fast with the increase of the applied voltage, and at $V_{\text {appl }}=7.0 \mathrm{~V}$ the response time $\tau_{r}$ was $238 \mathrm{~ms}$. On the other hand, for the traditional visible LC-SLM operating at $V_{\text {appl }}$ $=5.0 \mathrm{~V}$ the response time was $35 \mathrm{~ms}$, which is faster than that of the new LC-SLM. However, the $238 \mathrm{~ms}$ response time is an operative value for the feedback chirp compensation [2,3] of $1 \mathrm{kHz}$ to $100 \mathrm{MHz}$ repetitive femtosecond pulses.

In conclusion, for the first time to our knowledge, we developed a 1-pixel UV-LC-SLM operating in the

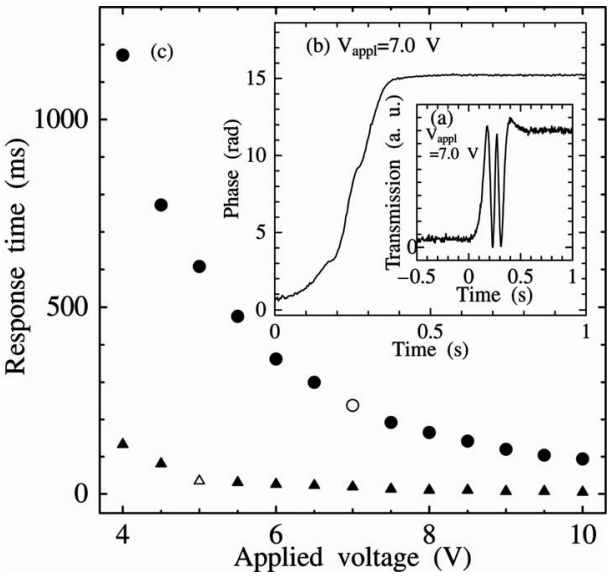

Fig. 4. Transient response of UV-LC-SLM at $633 \mathrm{~nm}$. (a) Transmitted light intensity $I(t)$ at $V_{\text {appl }}=7.0 \mathrm{~V}$. (b) Transient phase modulation calculated from $I(t)$ at $V_{\text {appl }}=7.0 \mathrm{~V}$. (c) Applied voltage $V_{\text {appl }}$ dependence of response time $\tau_{r}$ for UV-LC-SLM (open circles, $\tau_{r}$ at $7.0 \mathrm{~V}$ ) and visible LC-SLM (open triangles, $\tau_{r}$ at $5.0 \mathrm{~V}$ ).

spectral range from 260 to $1100 \mathrm{~nm}$ and clarified the phase modulation properties. The overall transmission efficiency is over $85 \%$. The UV-LC-SLM driving parameters were determined as $V_{\mathrm{DD}}=7.0 \mathrm{~V}$ and $T$ $=13 \mathrm{~ms}$. The amount of the phase modulation was much larger than $2 \pi$ rad at $1100 \mathrm{~nm}$. The response time was $238 \mathrm{~ms}$ at the applied voltage of $7.0 \mathrm{~V}$. Based on those results, very recently, we have fabricated a 648 pixel two-dimensional UV-LC-SLM. Using this multipixel UV-LC-SLM, we are now performing direct UV-to-NIR pulse shaping and high-power generation of single subcycle optical pulses. We believe that this will bring about a new field of research in coherent control of electrons in atoms, molecules, and solids by the electric-field force, including application for attosecond extreme-UV pulse generation.

\section{References}

1. A. M. Weiner, Rev. Sci. Instrum. 71, 1929 (2000).

2. M. Yamashita, K. Yamane, and R. Morita, IEEE J. Sel. Top. Quantum Electron. 12, 213 (2006).

3. E. Matsubara, K. Yamane, T. Sekikawa, and M. Yamashita, J. Opt. Soc. Am. B 24, 985 (2007).

4. N. Karasawa, R. Morita, H. Shigekawa, and M. Yamashita, Opt. Lett. 25, 183 (2000).

5. H. Rabitz, R. de Vivie-Eiedle, M. Motzkus, and K. Kompa, Science 288, 824 (2000).

6. V. Pervak, F. Krausz, and A. Apolonski, Opt. Lett. 32, 1183 (2007) and references therein.

7. F. Verluise, V. Loude, Z. Cheng, Ch. Spielmann, and P. Tournois, Opt. Lett. 25, 575 (2000).

8. A. Baltuška, T. Fuji, and T. Kobayashi, Opt. Lett. 27, 306 (2002)

9. M. Hacker, G. Stobrawa, R. Sauerbrey, T. Buckup, M. Motzkus, M. Wildenhain, and A. Gehner, Appl. Phys. B 76, 711 (2003).

10. K. Oka and T. Kato, Opt. Lett. 24, 1475 (1999). 